\title{
Mediterranean diet adherence and blood lipids in a very high cardiovascular risk group
}

\author{
T.J. Butler, K.J.J. Gabrysch, H.L. Tudball, A. Bhatkande and A. Woodall \\ Department of Clinical Sciences and Nutrition, University of Chester, Chester, CH1 4BJ, UK
}

The Mediterranean diet (MD) has been encouraged as a dietary pattern for health ${ }^{(1)}$ with the National Institute for Health and Care Excellence (NICE) recommending all individuals who have experienced a myocardial infarction (MI) follow such a diet ${ }^{(2)}$. However, previous work has suggested that older individuals in the UK struggle to follow this diet pattern ${ }^{(3)}$. The aim of the present study was to determine the level of adherence to a MD pattern in individuals deemed to be at very high risk of cardiovascular events. An additional aim was to identify if gender was a determinant of diet score and blood lipid concentrations.

Participants were sampled from 5 cardiac support groups across the North of the UK. Those meeting entry criteria of MI, coronary artery bypass graft, or percutaneous coronary intervention with or without stent were assessed for adherence to the MD using a validated 14-point questionnaire. Overall dietary intake was assessed using the EPIC-Norfolk food frequency questionnaire (FFQ). Capillary blood was analysed for triacylglycerol, total-cholesterol, low-density lipoprotein cholesterol, high-density lipoprotein-cholesterol and non-high-density lipoprotein cholesterol (TAG, TC, LDL-C, HDL-C and non-HDL-C, respectively). All procedures were approved by the faculty of medicine, dentistry and clinical sciences research ethics committee.

\begin{tabular}{|c|c|c|c|c|c|c|c|c|}
\hline \multirow[b]{2}{*}{ Group } & \multicolumn{2}{|c|}{ MDS } & \multicolumn{2}{|c|}{$\mathrm{TC}$} & \multicolumn{2}{|c|}{ LDL-C } & \multicolumn{2}{|c|}{ HDL-C } \\
\hline & Mean & SD & Mean & $\overline{\mathrm{SD}}$ & Mean & $\overline{\mathrm{SD}}$ & Mean & $\mathrm{SD}$ \\
\hline Total ( $n$ 65) & 6.6 & $2 \cdot 2$ & $4 \cdot 2$ & $0 \cdot 9$ & $2 \cdot 0$ & $0 \cdot 8$ & $1 \cdot 3$ & 0.4 \\
\hline Male $(n 40)$ & 5.9 & $2 \cdot 1$ & $4 \cdot 0$ & 0.8 & 1.9 & $0 \cdot 6$ & $1 \cdot 1$ & $0 \cdot 3$ \\
\hline Female ( $n$ 25) & 6.8 & $2 \cdot 0$ & 4.4 & 1.0 & $2 \cdot 2$ & 1.0 & $1.4^{*}$ & 0.5 \\
\hline
\end{tabular}

MDS, Mediterranean diet score; TC, total cholesterol; LDL-C low-density lipoprotein cholesterol; HDL-C, high-density lipoprotein-cholesterol and non-high density lipoprotein cholesterol Mean values for female HDL-C were significantly different from those for males (independent sample $t$ test): $* P<0 \cdot 05$.

Of the 236 eligible individuals, 65 met the inclusion criteria. Overall adherence to the MD pattern was poor, signified by a low MD score (MDS). Subgroup analysis revealed a trend for lower MDS in men compared to women $(P=0 \cdot 051)$. TC, TAG, LDL-C, and non-HDL-C did not differ between gender whereas HDL-C was significantly $(P<0.05)$ higher in women. There was a significant $(P<$ 0.05 ) effect of gender on participant intake of onion, leek or garlic and olive oil-flavoured sauces more than twice per week, with women more likely to adopt this diet component. Other components of the MD questionnaire did not differ between genders. Analysis of FFQ data revealed no significant difference between men and women.

In conclusion, these results suggest that adherence to the MD pattern for secondary cardioprotection is low in the North of the UK, with no difference between men and women.

This work was supported by the council for allied health professions research: Cheshire and Merseyside.

1. Gerber M \& Hoffman R (2015) Br J Nutr 113, S14-S10.

2. National Institute for Health and Care Excellence [NICE] (2013) Myocardial infarction: cardiac rehabilitation and prevention of further MI https://www.nice.org.uk/guidance/cg172 (accessed March 2016).

3. Lara J, Turbett E, Rudgard K et al. (2015) Maturitas 82, 387-393. 